\title{
Death by Caffeine: Presumptive Malicious Poisoning of a Dog by Incorporation in Ground Meat
}

\author{
S. N. Tawde • B. Puschner • T. Albin • S. Stump • \\ R. H. Poppenga
}

Published online: 27 October 2012

(C) American College of Medical Toxicology 2012

\begin{abstract}
Background A 4-year-old, $37 \mathrm{~kg}$, male German shepherd developed hyperthermia, tachycardia, and agitation following consumption of ground meat found in the backyard of its owner. When presented to a veterinary clinic, plasma ethylene glycol (EG) testing was positive, and the dog was given ethanol and lactated Ringer's solution intravenously. Approximately $11 \mathrm{~h}$ postexposure the dog died.

Discussion Among tissues submitted for toxicological analysis, urine was negative for $\mathrm{EG}$, ground meat was negative for certain drugs of abuse, and gastric contents were negative for zinc/aluminum phosphide and metaldehyde. Analysis of gastric contents by gas chromatography-mass spectrometry confirmed the presence of caffeine. Caffeine concentration in the ground meat was estimated at $1 \%$. Caffeine is a methylxanthine alkaloid with a reported canine oral median lethal dose $\left(\mathrm{MLD}_{50}\right)$ of $140 \mathrm{mg} / \mathrm{kg}$ (range 120 $200 \mathrm{mg} / \mathrm{kg}$ ). A commercially available 200 -mg tablet formulation of caffeine was considered to be a possible source but this was not confirmed. By conservative estimates, the dog would need to ingest approximately $500-550 \mathrm{~g}$ of the meat to reach the $\mathrm{MLD}_{50}$. Acute intoxication affects the cardiovascular, pulmonary, neurologic, gastrointestinal, and metabolic systems. Although no tablet remnants were observed in the bait, tablets could have been crushed and/or
\end{abstract}

A poster describing this case has been presented at the Society of Toxicology Annual Conference 2012, in San Francisco.

S. N. Tawde $(\bowtie) \cdot$ B. Puschner $\cdot$ S. Stump $\cdot$ R. H. Poppenga California Animal Health and Food Safety Laboratory,

School of Veterinary Medicine, University of California-Davis,

West Health Sciences Drive,

Davis, CA 95616, USA

e-mail: snehalt@rediffmail.com

T. Albin

Salazar Road Veterinary Clinic,

1025 Salazar Road,

Taos, NM 87571, USA dissolved. Other potential caffeine sources include guarana, brewed and concentrated coffee, and caffeine-containing beverages. Based on the history, clinical signs, and the detection of caffeine in the gastric contents and meat, a presumptive diagnosis of malicious caffeine poisoning was made. A suggested treatment regimen for caffeine intoxication in dogs is described. While few cases of accidental ingestion of caffeine by dogs have been described, the intentional use of a concentrated caffeine source to cause mortality in a dog has not been previously reported.

Keywords Caffeine $\cdot$ Poisoning $\cdot$ Bait $\cdot$ Ground meat

\section{Introduction}

The intentional use of caffeine to lethal effect in a dog has not been previously reported, although cases of accidental caffeine overexposure and subsequent mortality in dogs have been published [1-4]. Various caffeine-containing formulations can be easily obtained and incorporated in food attractive to animals. In cases of accidental caffeine overexposure, the clinical history may often reveal the source, examples of which include coffee/cacao bean products, chocolate and chocolate products, over-the-counter (OTC) stimulant tablets, medications, drinks, and herbal weight loss supplements containing guarana (Paullinia cupana) [1]. However, in cases of intentional poisoning, caffeine as a cause may not be readily suspected, unless awareness is increased among veterinarians and diagnostic professionals. Moreover, the clinical syndrome elicited by a lethal dose of caffeine may be attributed to a long list of stimulants, and valuable time and resources may be lost in eliminating and/ or addressing these suspicions.

Caffeine is a bitter-tasting methylated xanthine alkaloid. Theobromine is another methylxanthine that is found along with caffeine in chocolate/cacao products and is a metabolite 
of caffeine as well [5]. The concentration of theobromine is often higher than caffeine in several products [6]; therefore, theobromine is more commonly associated with intoxication as compared to caffeine, although they have similar toxic potential. Theophylline is another methylxanthine that is found along with caffeine in tea leaves and related decoctions. Although concentrated preparations of caffeine and theophylline represent a risk, animal poisonings have not been commonly reported [6]. The use of caffeine to intentionally poison a dog via incorporation in ground meat is unusual. This report describes the clinical signs, exposure assessment, and toxicological findings in a dog suspected to have died from caffeine.

\section{Case Presentation}

The owner saw his $37 \mathrm{~kg}$, 4-year-old male-castrated German shepherd eating a cooked ground meat patty in the backyard. As he had not provided the meat patty, the owner became suspicious and tried to stop the dog; however, a significant portion had been consumed. A very small portion of the meat remained $(<5 \mathrm{~g})$ and was collected by the owner. The dog became excitable and was presented to a veterinary clinic $3 \mathrm{~h}$ postexposure with tachycardia (140-150, normal 70-120 beats per min (bpm) [7]), hyperthermia (40 ${ }^{\circ} \mathrm{C}$, normal $38.9{ }^{\circ} \mathrm{C}$ [7]), polydipsia, and agitation. Hematological and serum biochemical parameters, including serum osmolality, were within acceptable or nondiagnostic ranges. Serum testing for ethylene glycol (EG) using a commercially available kit ${ }^{1}$ at the clinic suggested the presence of EG, but not at "toxic" concentrations. At the veterinarian's discretion, the dog was administered $20 \%$ ethanol at $5.4 \mathrm{ml} / \mathrm{kg}$ intravenously, diluted in lactated Ringer's solution, twice during the course of treatment, at approximately 4 and $8 \mathrm{~h}$ postexposure via the cephalic vein. During treatment, the patient continued to be agitated and removed the intravenous catheter. Six hours postexposure, acepromazine was administered intravenously at $0.02 \mathrm{mg} / \mathrm{kg}$, which provided some relaxation. Diarrhea occurred once, approximately $7 \mathrm{~h}$ postexposure. Based on a history of suspected drug and/or gang-related activity in the neighborhood of the dog, the urine was tested at the veterinary clinic using a home-use drug testing $\mathrm{kit}^{2}$ and tested negative for the presence of as amphetamine, methamphetamines, marijuana, cocaine, and opiates. Tachycardia continued (180 bpm) and the body temperature returned to normal $\left(39^{\circ} \mathrm{C}\right)$ without additional therapy. In another hour, the cardiac rate was noted to be $150-160 \mathrm{bpm}$, and the patient was resting more comfortably. However, approximately $11 \mathrm{~h}$

\footnotetext{
${ }^{1}$ Sources and manufacturers: PRN React EG test kit, PRN Pharmacal, Pensacola, FL 32514

${ }^{2}$ Sources and manufacturers: At Home Drug Test, PharmaTech Inc., San Diego, CA 92121
}

postexposure, the patient was found dead in his kennel. On gross necropsy, the stomach was noted to be full and contain undigested ground meat pieces. Along with the previously collected ground meat patty $(<5 \mathrm{~g})$, samples of stomach content, urine, liver, and kidneys were submitted for toxicological analysis.

At the request of the owner, the stomach content was tested for zinc and aluminum phosphide, which are a rodenticide and a grain fumigant, respectively. Both compounds release the poisonous gas phosphine in the acid environment of the carnivore stomach. The gastric contents tested negative for both agents at a reporting limit of $1 \mathrm{mg} / \mathrm{kg}$. The urine tested negative for EG at a reporting limit of $5 \mathrm{mg} / \mathrm{kg}$. Since, gang and drug-related activity was reported in the owner's neighborhood, screening for illicit drugs was performed on the ground meat sample. The following drugs of abuse were not detected: amphetamine, methamphetamine, phentermine, cocaine, ephedrine, lysergic acid diethylamide, nicotine, psilocin, tetrahydrocannabinol, methadone, and morphine. Based on the initial increase in body temperature and agitation, metaldehyde (snail bait) use was suspected by the treating veterinarian. During gas chromatography-mass spectroscopy (GC-MS) screening for metaldehyde, the stomach contents tested negative for metaldehyde at a reporting limit of $0.1 \mathrm{mg} /$ $\mathrm{kg}$; however, caffeine was detected through mass spectral matching. Since this was not a targeted analysis for caffeine, the stomach contents were qualitatively re-analyzed. Based on a match in retention time and mass spectrum with the caffeine standard (Fig. 1), the presence of caffeine was confirmed in stomach contents. Due to financial considerations expressed by the client, the caffeine concentration of the gastric contents was not determined; instead, a confirmatory analysis on the meat patty was requested and undertaken, to investigate the source of caffeine. The ground meat sample was similarly analyzed and was found to contain approximately $1 \%$ caffeine.

\section{Discussion}

The malicious use of caffeine to lethal effect can be challenging to treat as well as to detect and confirm. The presentation of a hyperthermic, agitated patient with tachycardia requires quick assessment and action on part of the veterinarian. Based on the history and reported clinical signs exhibited by the dog, the list of differential diagnosis can be extensive, and a mass screening-based approach as compared to specific diagnostic testing may prove to be time and cost effective. A clear history or timely diagnostic support may not always be available, compounded by the limited financial resources of many animal owners. Considering these limitations, it would be useful to review the clinical risk assessment and compare the findings 
Fig. 1 Selected ion

chromatogram and retention time of a $10-\mu \mathrm{g} / \mathrm{ml}$ caffeine standard compared with the large peak of caffeine in the stomach contents of the dog

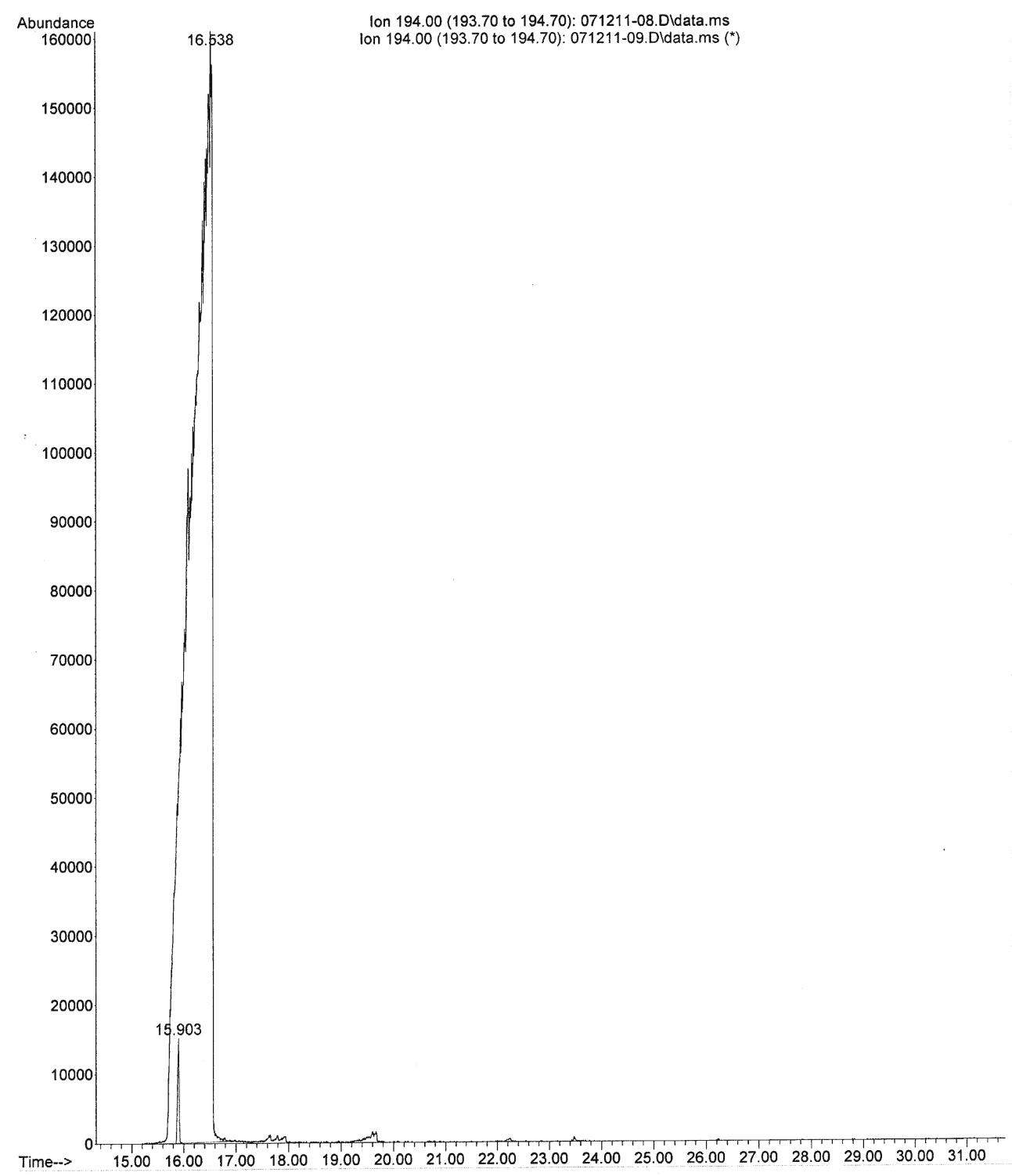

in this case with examples of previously reported cases of accidental caffeine exposure. The serendipitous detection and confirmation of caffeine is equally interesting. In addition, the treatment for caffeine intoxication is discussed.

The detection and analytical confirmation of caffeine in the gastric contents and the meat patty necessitates further evaluation, to determine if caffeine may be responsible for the clinical signs and death of the dog. The lethal dose of caffeine in the dog varies from 110 to $200 \mathrm{mg} / \mathrm{kg}$ of body weight, and the median lethal dose $\left(\mathrm{MLD}_{50}\right)$ for dogs is reported to be $140 \mathrm{mg} / \mathrm{kg}$ body weight [6]. At an approximate concentration of $1 \%$ caffeine in the meat, the dog would need to ingest approximately 500-550 g of bait to reach the $\mathrm{MLD}_{50}$, assuming that caffeine is uniformly distributed in the bait. The formulation of bait with sufficient caffeine to kill a dog can be challenging, unless a purified source is readily available. With that consideration, non- prescription-grade OTC caffeine pills were considered a possible readily available source. Approximately 26 pills of $200 \mathrm{mg}$ each could be lethal for a dog weighing $37 \mathrm{~kg}$. Visual examination of the meat sample did not reveal any pills/pill fragments. It is possible that the pills were crushed and allowed to dissolve in the meat. As previously stated, the consumption of a significant portion of the meat patty (containing approximately $1 \%$ caffeine) combined with the observation of a stomach filled with undigested ground meat pieces supports the likelihood that caffeine may have been delivered at a lethal dose. The weight of the meat patty and that of the stomach contents is not known.

The pharmacological and toxicological effects of caffeine are mediated via several mechanisms: (1) the nonselective antagonism of adenosine receptors, (2) the inhibition of cyclic nucleotide phosphodiesterase, (3) the release of calcium from intracellular stores, and (4) antagonism of benzodiazepine 
receptors [8]. At higher doses not typically consumed, it can cause gamma-aminobutyric acid inhibition due to the blocking of $\mathrm{GABA}_{\mathrm{A}}$ receptors $[9,10]$. The ability of caffeine to competitively inhibit adenosine receptors plays a central role in its stimulatory effect on behavior and cognitive function [11]. The cardiostimulatory effects of caffeine are thought to be due to the inhibition of phosphodiesterases [11]. Caffeine intoxication leads to a positive inotropic, chronotropic, and dromotropic [12] effects on the heart, cerebral vasoconstriction, renal vasodilation, smooth muscle relaxation in the gastrointestinal tract, and stimulation of gastric secretion [1]. It should be noted from this and other reports [1-4] that the bitter taste of caffeine does not deter dogs from consuming it, although the masking of bitterness by the delivery vehicle or other included ingredients may occur.

Caffeine is rapidly and completely absorbed, with $99 \%$ being absorbed within 45 min of ingestion [11]. Peak serum concentrations are reached in 30-60 min, and clinical signs of an overdose may be observed in dogs within $2 \mathrm{~h}$ [2] postingestion.

Cases of accidental caffeine exposure and intoxication in dogs have been previously reported. A 2-year-old, $11 \mathrm{~kg}$ poodle with caffeine intoxication developed hyperirritability, hypersensitivity to touch, sudden rigidity accompanied by a single tetanic convulsion, and tachycardia prior to death [3]. The dog had consumed white caffeine tablets which appeared to be crudely made and fragile. Forensic analysis confirmed that the tablets were pure caffeine and it was speculated that the tablets were street drugs sold as amphetamine substitutes for unsuspecting buyers. Another report [2] describes consumption of $3 \mathrm{~g}$ caffeine by a $7-\mathrm{kg}$ dog, which in $2 \mathrm{~h}$ gave rise to a semi-tetanic state, hyperthermia $\left(40{ }^{\circ} \mathrm{C}\right)$, rapid and weak pulse, dilated pupils, and sluggish reflexes followed by death. A report [4] of a terrier-type dog that had swallowed commercially available OTC caffeine tablets describes excitability, a heart rate of $>215$ followed by death in $5 \mathrm{~h}$. Thus, hyperexcitability, hyperthermia, and tachycardia are commonly observed. While not all clinical signs may have been described in previously reported cases, or noted in the current case, a comparatively longer period of illness was noted in this case. Based on the available data, identifying a probable cause for the longer period of illness may not be possible; however, it can be speculated that the differential kinetic release of caffeine from the meat patty and/or the dose of caffeine may be primarily responsible. Vomiting and abdominal pain are frequently described toxic effects of caffeine in humans [13]. However, those have not been reported in animal cases of intoxication. The immediate physiological cause/s of death (e.g., cardiac failure or tetanic seizure) was not determined, in this case. The most common cause of death due to caffeine intoxication in humans is ventricular fibrillation, which has been replicated in anesthetized rats [13].
If metaldehyde testing using GC-MS had not been performed, the serendipitous detection of caffeine in the stomach contents would not have occurred. This is a good example of the utility of GC-MS to identify a wide range of potential toxicants in a variety of sample matrices based upon mass spectral matches of unknown compounds with mass spectral libraries. Since theobromine was not detected in the meat or stomach contents of the dog, chocolate or cacao bean products as a source of caffeine can be ruled out. No data regarding sensitivity or specificity of the ethylene glycol $^{3}$ or the home drug test $\mathrm{kit}^{4}$ used at the veterinary clinic were identified in published scientific literature.

There is no antidote to caffeine, and treatment is based on clinical signs. In confirmed cases of exposure that has occurred within $2 \mathrm{~h}$, and no clinical signs of caffeine intoxication are evident, emesis should be induced with $3 \%$ hydrogen peroxide $(1.5 \mathrm{ml} / \mathrm{kg}, \mathrm{PO})$ or apomorphine $(0.02$ to $0.04 \mathrm{mg} / \mathrm{kg}$, IM or IV). Multidose activated charcoal (AC) administration at $0.5 \mathrm{~g} / \mathrm{kg}$ orally or by stomach tube every $3 \mathrm{~h}$ for $72 \mathrm{~h}$ is recommended during treatment for methylxanthine (caffeine and theobromine) intoxication, due to entero-hepatic recirculation [6]. Additionally, a cathartic (e.g., sorbitol) may be administered with the first dose of $\mathrm{AC}$, to enhance the excretion of caffeine. Whether multidose AC administration with or without a cathartic may have made a difference to this patient is debatable, considering the rapid absorption of caffeine and the short time needed to reach peak plasma concentration. If the patient's condition contraindicates the induction of emesis (e.g., presence of CNS excitation/depression or extreme tachycardia), gastric lavage should be performed. The use of a cuffed endotracheal tube will decrease the risk of aspiration, and a dose of activated charcoal with a cathartic should be left in the stomach after lavage. The early suspicion of EG intoxication based upon a false positive point-ofcare test might have led to a decision not to administer AC. The reason is that $\mathrm{AC}$ is considered to be of limited benefit for the treatment of EG intoxication due to rapid absorption of ethylene glycol from the gastrointestinal tract and limited adsorption to AC. Ethanol was chosen over fomepizole as an antidote to ethylene glycol due to cost considerations. The dose of ethanol administered to the patient followed a recommended guideline [14] and did not rapidly cause CNS depression, therefore ethanol was not considered to be a cause/factor of death in this dog. Side effects associated with ethanol administration include apnea and intravascular hemolysis [15]. Apnea was not observed in this dog.

Stabilization of dogs with clinical signs such as hyperactivity, tremors, seizures, or tachycardia is necessary before attempting detoxification. Anticonvulsant therapy may be

\footnotetext{
${ }^{3}$ Please refer to footnote 1

${ }^{4}$ Please refer to footnote 2
} 
utilized in stabilizing patients with CNS signs. Hyperexcitability, shaking, signs of nervousness, tremors, or seizures may be controlled with phenothiazine tranquilizers such as acepromazine ( 0.05 to $1.0 \mathrm{mg} / \mathrm{kg}$ IV, IM, or SC). Alternatively, diazepam can be utilized as a $0.5-1.0-\mathrm{mg} / \mathrm{kg}$ slow IV bolus, which may be repeated as necessary two to three times at 5-10 min intervals. If pseudoephedrine intoxication is on the list of differentials, then diazepam use should be avoided, since dissociative effects of benzodiazepines like diazepam are generally exaggerated in pseudoephedrine intoxication [1]. Another drug for controlling seizures is phenobarbital at 3-4 mg/kg IV, titrated to effect. Sodium pentobarbital is generally reserved for the treatment of uncontrollable seizures in dogs, when diazepam and phenobarbital have failed. It can be administered at $5-20 \mathrm{mg} / \mathrm{kg}$ $\mathrm{IV}$, titrated to effect, as a general anesthetic to stop motor activity. If CNS signs are uncontrolled from the preceding measures, administration of gas anesthetic such as isoflurane may be useful [1]. Metoprolol is preferred over propranolol for the control of tachycardia, since metoprolol does not slow renal excretion of methylxanthines like caffeine, as propranolol can [16]. A suggested starting dose of either metoprolol or propranolol is $0.02-0.06 \mathrm{mg} / \mathrm{kg}$, slow IV, every $6 \mathrm{~h}$. Body temperature, blood pressure, and electrocardiographic tracings should be monitored. After stabilization of adverse CNS and cardiovascular signs, fluid diuresis should be used to enhance caffeine excretion, as it is primarily eliminated through the kidneys. Methylxanthines and their metabolites can be re-absorbed across the bladder wall; therefore, placement of a urinary catheter is useful. Clinical signs may persist up to $72 \mathrm{~h}$ in severe cases [16]. Fluid and electrolyte changes should be monitored and corrected as needed. Baseline serum electrolyte concentrations should be determined, and serum biochemical analyses should be performed and repeated in 2-3 days. For a patient that survives, treatment and monitoring should continue until all clinical signs of intoxication have resolved.

Although the referring veterinarian performed a necropsy and no obvious lesions were noted, the carcass was neither examined by a board-certified veterinary pathologist nor histopathological investigations undertaken. A thorough necropsy is generally warranted to help identify any underlying and undiagnosed causes as reasons of death. However, the young age of the dog with no previously known health issues, consumption of caffeine-containing bait, and clinical signs consistent with caffeine intoxication lead to a presumptive diagnosis of caffeine intoxication. The offering of cooked meat from an unknown source strongly suggests malicious intent. The lack of any other toxicological findings despite a reasonably extensive investigation supports this diagnosis, although it is possible that other compounds not detected by GC-MS could be present in the meat patty.

Acknowledgments The authors thank Mike Filigenzi and Marcia Booth for laboratory assistance.

Conflict of Interest The authors have no conflict of interest to declare.

\section{References}

1. Ooms TG, Khan SA, Means C (2001) Suspected caffeine and ephedrine toxicosis resulting from ingestion of an herbal supplement containing guarana and ma huang in dogs: 47 cases (19971999). J Am Vet Med Assoc 218(2):225-229

2. Wigderson FJ (1956) Accidental caffeine poisoning in a dog. J Am Vet Med Assoc 129:233

3. Foor J, Stowe C (1975) Acute fatal caffeine toxicosis in a dog. J Am Vet Med Assoc 167(5):379

4. Michael SJ (1961) Caffeine poisoning in a dog. J Am Vet Med Assoc 139:559

5. Eteng $M$ et al (1997) Recent advances in caffeine and theobromine toxicities: a review. Plant Foods Hum Nutr (Formerly Qualitas Plantarum) 51(3):231-243

6. Carson TL (2006) Methyxanthines. In: Peterson ME, Talcott PA (eds) Small animal toxicology. Saunders/Elsevier, St. Louis, pp $845-851$

7. Detweiler DK (2011) Reference guides: Dukes' physiology of domestic animals, in Merck veterinary manual. Merck, Whitehouse Station

8. Myers JP, Johnson DA, McVey DE (1999) In: Gupta BS, Gupta U (eds) Caffeine and the modulation of brain function, in caffeine and behavior: current views and research trends. CRC, Boca Raton, pp 17-30

9. Pelchovitz DJ, Goldberger JJ (2011) Caffeine and cardiac arrhythmias: a review of the evidence. Am J Med 124(4):284-289

10. Fredholm BB, Bättig K, Holmén J, Nehlig A, Zvartau EE (1999) Actions of caffeine in the brain with special reference to factors that contribute to its widespread use. Pharmacol Rev 51(1):83-133

11. Fernstrom JD (2001) Pharmacology of caffeine. In: VanDerVeen JE (ed) Caffeine for the sustainment of mental task performance: formulations for military operations. Committee on Military Nutrition Research, Food and Nutrition Board, Institute of Medicine, Washington, pp 25-32

12. Benthe HF (1954) Dromotropic effect of caffeine on heart muscle of frog as a potassium sensitization. Naunyn-Schmiedebergs Arch Exp Pathol Pharmakol 223(4):285-293

13. Holmgren P, Nordén-Pettersson L, Ahlner J (2004) Caffeine fatalities - four case reports. Forensic Sci Int 139(1):71-73

14. Thrall MA, Connally HE, Grauer GF, Hamar D (2006) Ethylene glycol. In: Peterson ME, Talcott PA (eds) Small animal toxicology. Saunders/Elsevier, St. Louis, pp 702-726

15. Claus MA, Jandrey KE, Poppenga RH (2011) Propylene glycol intoxication in a dog. J Vet Emerg Crit Care 21(6):679-683

16. Kovalkovičová N, Šutiaková I, Pistl J, Šutiak V (2009) Some food toxic for pets. Interdiscip Toxicol 2(3):169-176 